EPJ Web of Conferences 42, 04003 (2013)

DOI: $10.1051 /$ epjconf/20134204003

(C) Owned by the authors, published by EDP Sciences, 2013

\title{
A Monte Carlo Simulation of Prompt Gamma Emission from Fission Fragments
}

\author{
D. Regnier ${ }^{1, a}$, O. Litaize ${ }^{1}$, and O. Serot ${ }^{1}$ \\ ${ }^{1}$ CEA, DEN, DER, F-13108 Saint Paul Lez Durance, France
}

\begin{abstract}
The prompt fission gamma spectra and multiplicities are investigated through the Monte Carlo code FIFRELIN which is developed at the Cadarache CEA research center. Knowing the fully accelerated fragment properties, their de-excitation is simulated through a cascade of neutron, gamma and/or electron emissions. This paper presents the recent developments in the FIFRELIN code and the results obtained on the spontaneous fission of ${ }^{252} \mathrm{Cf}$. Concerning the decay cascades simulation, a fully Hauser-Feshbach model is compared with a previous one using a Weisskopf spectrum for neutron emission. A particular attention is paid to the treatment of the neutron/gamma competition. Calculations lead using different level density and gamma strength function models show significant discrepancies of the slope of the gamma spectra at high energy. The underestimation of the prompt gamma spectra obtained regardless our de-excitation cascade modeling choice is discussed. This discrepancy is probably linked to an underestimation of the post-neutron fragments spin in our calculation.
\end{abstract}

\section{Introduction}

The conception of new designed PWR with massive steel reflector made the gamma heating problematic a burning issue. In order to predict this heating, coupled neutron-photon transport calculation based on gamma production evaluated data must be performed. Concerning the fission reaction, the prompt gamma multiplicity $\left(M_{\gamma}\right)$ and the prompt gamma spectrum $\left(N_{\gamma}\left(\epsilon_{\gamma}\right)\right)$ are required. Their evaluations which can be found in the last JEFF-3.1.1 and ENDF/B-VII releases for the induced fission of ${ }^{235} \mathrm{U}$ and ${ }^{239} \mathrm{Pu}$ and the spontaneous fission of ${ }^{252} \mathrm{Cf}$ have been made in the early 70's [1]. They are based on the same experiment realized by Verbinski [2]. Moreover, these spectra are assumed to be independent of the incident neutron energy and have been used to fulfill other isotopes evaluated files such as ${ }^{238} \mathrm{U},{ }^{233} \mathrm{U},{ }^{241} \mathrm{Pu}$. These lacuna in the evaluated library motivate us to study more deeply the fission fragment de-excitation process through the Monte Carlo code FIFRELIN [3, 4] which is developed at the Cadarache CEA research center.

In this paper, two different models implemented to simulate the de-excitation process of the fragments are exposed. The prompt gamma multiplicity and spectra coming from these simulations are compared and discussed. Finally, the discrepancies between simulation using different models of level density $(\rho(E, J, \pi))$ and gamma strength function $\left(f_{X L}\left(\epsilon_{\gamma}\right)\right)$ are highlighted as well as the influence of a variation of the fragments total angular momentum.

\footnotetext{
ae-mail: david.regnier@cea.fr
} 


\section{Model 1: Uncoupled neutron and gamma emission}

\subsection{Model description}

The first model of decay cascade that have been implemented in the FIFRELIN code is based on the assumption that all the prompt neutrons are emitted before the prompt gamma and the conversion electrons. While the excitation energy of the fission fragment is higher than a limit energy, a neutron is emitted with its kinetic energy in the fragment frame sampled from a Weisskopf spectrum. The limit energy is determined as follows:

$$
E_{\text {limit }}=S_{n}+E_{\text {rot }}(J), \quad E_{\text {rot }}=\frac{J(J+1) \hbar^{2}}{2 k \mathcal{J}_{\text {rig }}}
$$

where the rotational energy $E_{r o t}(J)$ is a function of the spin $J$ of the fragment, and of a fraction $k$ of its rigid body moment of inertia $\mathcal{J}_{\text {rig }}$. It is assumed that each neutron emission decreases the total angular momentum of the fragment by $\hbar / 2$.

When the limit energy is reached, the gamma decay takes place. Its simulation is based on the knowledge of a complete level scheme of the post-neutron fragment up to a given continuum beginning energy. This energy is chosen so that the level density is equal to $10^{5}$ levels per MeV. Below that continuum energy, a complete discrete level scheme is determined using a process already described in Ref. [4]. Experimental levels coming from the RIPL-3 [5] database are taken into account. Above the continuum beginning energy, the detail of discrete levels is no more computed and the decay algorithm relies on average nuclear properties over $1 \mathrm{keV}$ energy bins. Knowing the level scheme and the initial excited level $i$ of the post-neutron fragment, the code is able to calculate the decay probability of every possible transitions to a reachable level or bin $f$. These probabilities are either given by experimental data from RIPL-3 or in most cases calculated using the partial gamma width of the initial level. For a discrete final level $f$, this probability can be written:

$$
I_{\gamma}(i \rightarrow f, X L)=\frac{\Gamma_{\gamma}(i \rightarrow f, X L)}{\Gamma_{\gamma, \text { tot }}}, \quad \Gamma_{\gamma}(i \rightarrow j, X L)=\frac{f_{X L}\left(\epsilon_{\gamma}\right) \epsilon_{\gamma}^{2 L+1} y_{\text {fluctuation }}}{\rho\left(E_{f}, J_{f}, \pi_{f}\right)},
$$

where $y_{\text {fluctuation }}$ accounts for the Porter-Thomas fluctuation and is sampled as described in Ref. [6]. The probability to reach a final bin in the continuum region is obtained by integrating Eq. 1 over the final bin width. Based on this probability distribution, a transition is sampled and a new excited level or bin is reached. The same algorithm is repeated until a stable or metastable level of the fragment is reached.

\subsection{Results}

A simulation of the ${ }^{252} \mathrm{Cf}$ spontaneous fission has been performed using tabulated values of level densities taken from the RIPL-3 microscopic data as well as an enhanced generalized Lorentzian strength function. The average total energy dissipated as gamma-rays $\left(E_{\gamma, t o t}\right)$ is found to be $8.1 \mathrm{MeV}$ whereas the average gamma multiplicity is $8.0 \mathrm{\gamma} / \mathrm{f}$. These results were obtained from $4.10^{5}$ fission events so that the statistical error is less than $0.1 \%$ for these quantities. In order to compare these results with experimental data, the energy threshold in gamma detection must be taken into account. Several experimental data obtained with a detection energy threshold between 100 and $200 \mathrm{keV}$ have been reported in Table 1 . The corrected values of multiplicities and total gamma energy coming from FIFRELIN have been calculated without taking into account gamma-rays under $150 \mathrm{keV}$. The Fig. 1 


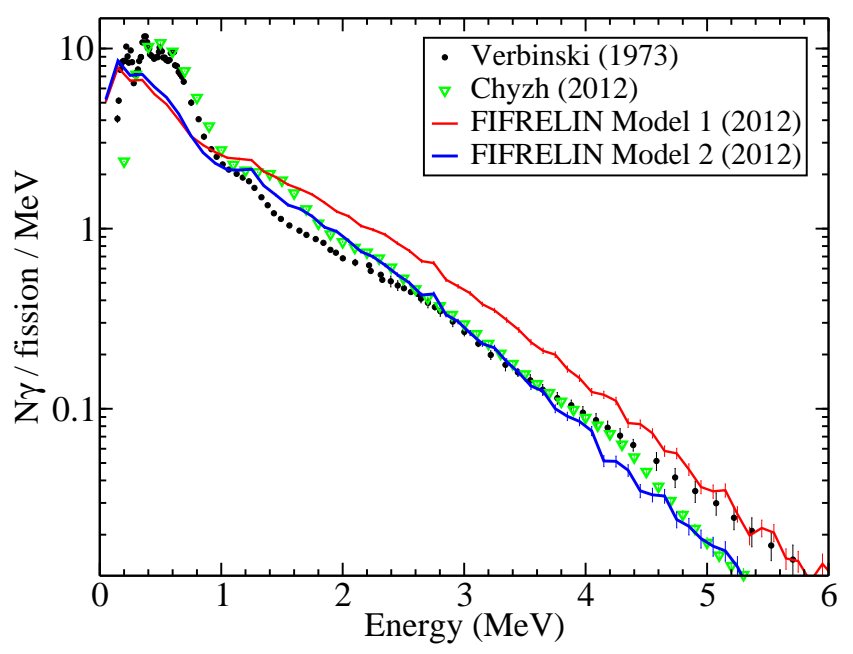

Figure 1. Total prompt gamma spectrum from the ${ }^{252} \mathrm{Cf}$ spontaneous fission

shows that the total prompt gamma spectrum obtained with that model is harder than experimental spectra.

In the low energy region, the spectrum in the fragment frame calculated with the same energy resolution as the Verbinski experiment emphasizes much more structures (cf. Fig. 2). This can be explained by the Doppler effect which must be taken into account to reproduce the experimental conditions. Assuming in a first approximation that the gamma emission is isotropic in the fragment frame and that the fragments are not losing any speed in the sample, one can derive the gamma emission spectrum in the laboratory frame. The structures obtained for the laboratory spectrum are more consistent with experiment.

Table 1. Observables from spontaneous fission of ${ }^{252} \mathrm{Cf}$

\begin{tabular}{lllll}
\hline Reference & $M_{\gamma}(\gamma / \mathrm{f})$ & $E_{\gamma, t o t}(\mathrm{MeV} / \mathrm{f})$ & $\bar{v}(\mathrm{n} / \mathrm{f})$ & Date \\
\hline Smith et al. [7] & 10.3 & 8.2 & & 1956 \\
\hline Verbinski et al. [2] & 7.80 & $6.84 \pm 0.3$ & & 1973 \\
\hline Nardi et al. [8] & $/$ & $6.7 \pm 0.4$ & & 1973 \\
\hline Skarsvag [9] & $9.76 \pm 0.4$ & $6.99 \pm 0.3$ & & 1980 \\
\hline Chyzh et al. [10] & $8.16 \pm 0.4$ & $(7.8)$ & & 2012 \\
\hline Vorobyev et al. [11] & & & $3.76 \pm 0.03$ & 2004 \\
\hline FIFRELIN Model 1 corrected & 7.1 & 8.0 & 3.78 & 2012 \\
\hline FIFRELIN Model 2 corrected & 6.6 & 6.3 & 4.05 & 2012 \\
\hline
\end{tabular}

\subsection{Parameters and models variation}

The prompt gamma spectrum has been calculated using several empirical formula as well as microscopic calculation results for the level densities and the radiative strength functions. Implemented 

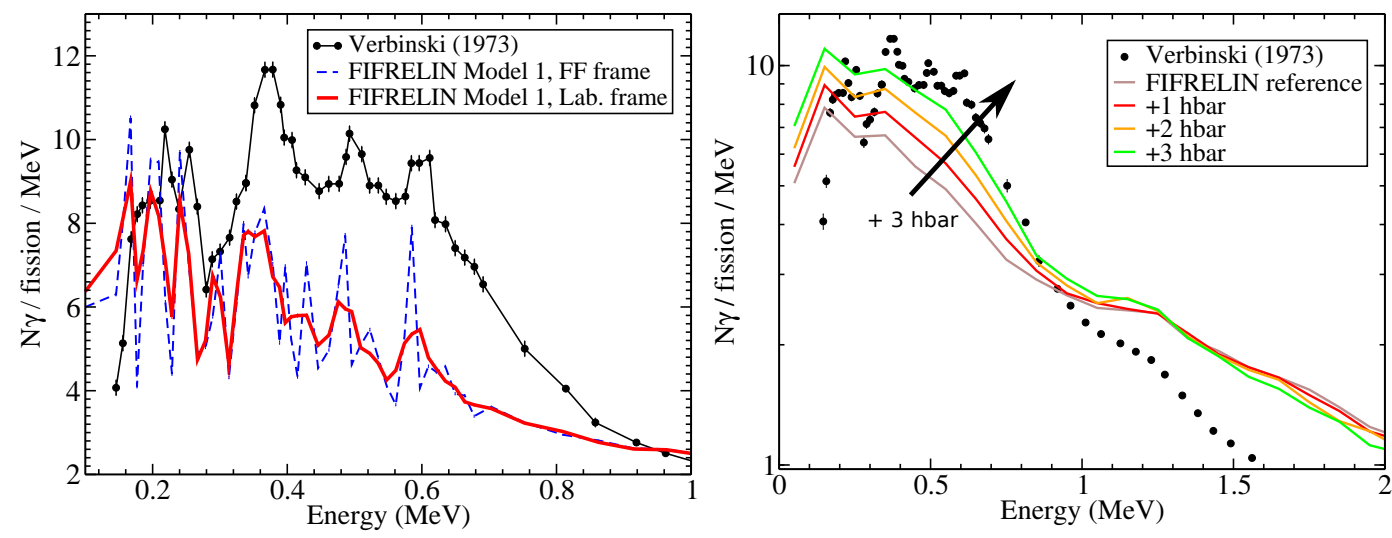

Figure 2. Low energy part of the prompt gamma spectrum for the spontaneous fission of ${ }^{252} \mathrm{Cf}$ : (left side) Impact of the fragment velocity; (right side) Effect of an artificial increase of the post-neutron fragment spin.

models for the level density are the constant temperature model, the composite Gilbert-Cameron model, and RIPL-3 tabulated values provided by HFB calculation. The strength function models used were the standard Lorentzian, the enhanced generalized Lorentzian and also tabulated values coming from microscopic calculation. Changing these models modifies the slope of the high energy part of the spectrum but does not affect the gamma-ray multiplicity below $0.8 \mathrm{MeV}$. Therefore, the underestimation of the gamma spectrum in that low energy range cannot be explained by the discrepancy between these models.

During the last stage of the gamma ray cascade, the gamma emission is imposed by the Yrast line, and therefore must be highly sensitive to the ratio between the excitation energy and the total angular momentum of the post neutron-fragment. Several studies $[9,12,13]$ on the total angular momentum distribution of the fragments have been carried out but their results are based on the study of even-even post neutron fragments and a $2 \hbar$ uncertainty is associated to their average spin value. In FIFRELIN, the spins of the primary fragments are sampled in an empirical law:

$$
P(J)=\frac{(J+1 / 2)}{\sigma^{2}(T)} e^{-\frac{(J+1 / 2)^{2}}{2 \sigma^{2}(T)}}
$$

where the spin cut-off $\sigma^{2}(T)$ can be temperature dependent or constant. The average spin values obtained with a temperature dependent spin cut-off are around $7 \hbar$ and $6 \hbar$ for the heavy and light fission fragment respectively. Moreover the hypothesis of a $\hbar / 2$ decrease of the fragment total angular momentum at each neutron emission leads to lower values for the post-neutron spin. These assumptions can be discussed, and are probably the cause of the underestimation of the calculated low gammarays multiplicity. To illustrate this assertion, we artificially increased the value of both post-neutron fragments spin. As can be seen on Fig. 2, this leads to an increase of the low energy gamma ray multiplicity.

\section{Model 2: Coupled neutron and gamma emission}

Even if the first model enables us to calculate a rough prompt gamma spectrum, it relies on several hypothesis that can be discussed such as the average variation of the fragments angular momentum 
during a neutron emission. This motivates the implementation of another de-excitation model that is free of these hypothesis.

\subsection{Description of the model}

The basic idea, in order to take into account the neutron/gamma competition, is to simulate the decay cascade in the frame of the Hauser-Feshbach statistical model [14]. Both neutron and gamma emission are considered possible from the beginning of the de-excitation and the probability to decay from the level $i$ to a reachable level $f$ is given by the partial width of the transition $(\Gamma(i \rightarrow f))$ :

$$
p(i \rightarrow f, X L \text { or } l, j)=\frac{\Gamma(i \rightarrow f, X L \text { or } l, j)}{\Gamma_{\gamma}^{\text {tot }}+\Gamma_{\text {neutron }}^{\text {tot }}}
$$

The gamma partial width are calculated as previously described. If the neutron emission channel is open, the neutron partial width is given by the formula:

$$
\Gamma_{n}(i \rightarrow f, l, j)=\frac{T_{l, j}\left(\epsilon_{n}\right) y_{\text {fluctuation }}}{2 \pi \rho\left(E_{f}, J_{f}, \pi_{f}\right)}
$$

where $T_{l, j}\left(\epsilon_{n}\right)$ are the neutron transmission coefficients provided by a TALYS-1.4 [15] optical model calculation using the Koning-Delaroche spherical potential.

\subsection{Results and discussion}

With the model 2, the number of gamma rays emitted before the last prompt neutron is calculated in order to check the validity of the hypothesis made in the first model. It is found that in average, such a gamma is emitted once every 250 fission events.

The part of the excitation energy dedicated to the neutron emission is increased by nearly $2 \mathrm{MeV}$ compared with the model 1 , whereas the average neutron energy in the fragment frame is decreased by $0.1 \mathrm{MeV}$. The average decrease of spin due to one neutron emission is calculated and evaluated to be $0.1 \hbar$ per neutron. This leads to an increase of the post-neutron fragment spin of about $0.9 \hbar$ for both light and heavy fragments compared to the model 1 .

The total excitation energy dissipated as gamma rays is lower than the one obtained through the model 1 (see Table 1). However, due to the increase of the spin over excitation energy ratio of the post-neutron fragments, the average gamma energy is evaluated to be $0.86 \mathrm{MeV}$ instead of $1.02 \mathrm{MeV}$ for the model 1. The spectrum shown in Fig. 1 is more consistent with experimental data but still underestimated in the low energy region.

\subsection{Parameters and models variation}

A preliminary study of the impact of the level density and gamma strength function models as well as variations of the primary fragments spin has been realized. The choice of the level density model implies variation of few per cents on the neutron multiplicity, the total gamma energy as well as the average gamma multiplicity. The simulations performed with different gamma strength function models show variations of the neutron observables and the total gamma energy lower than $1 \%$. A more important variation of $12 \%$ is observed on the average gamma energy. Last but not least, an increase of $2 \hbar$ on the average angular momentum of primary fission fragments favores the gamma emission by increasing the average total gamma energy by $0.7 \mathrm{MeV}$. This modification increases significantly the gamma ray multiplicity in the low energy part of the gamma spectrum leading to a $7 \%$ decrease of the average gamma energy. 


\section{Conclusion}

In that work, the two main fragment de-excitation models implemented in the FIFRELIN code are described. The second model, based on a Hauser-Feschbach treatment of the de-excitation, requires no more hypothesis on the evolution of the fragment spin during the neutron emission. Moreover, this model provides a better description of the neutron/gamma competition. The gamma observables obtained with the two different models are compared to experimental data and we show that the underestimation of the gamma spectrum predicted by the two models is probably due to an underestimation of the post-neutron fragments total angular momentum. The level density and strength function models used for our decay cascade simulations rules the slope of the high energy part of the gamma spectrum.

Among the numerous perspectives to this work, we consider testing the neutron emission using several optical model potentials. Finally, improvements of the description of the angular momentum distribution of the primary fission fragments must be carried on.

\section{References}

[1] R.E. Hunter, L. Stewart, Tech. Rep. LA-4901 (1972)

[2] V.V. Verbinski, H. Weber, R.E. Sund, Phys. Rev. C 7, 1173 (1973)

[3] O. Litaize, O. Serot, Phys. Rev. C 82, 054616 (2010)

[4] D. Regnier, O. Litaize, O. Serot, Phys. Proc. 31, 59 (2012)

[5] R. Capote, M. Herman, P. Oblozinsky, P. Young, S. Goriely, T. Belgya, A. Ignatyuk, A. Koning, S. Hilaire, V. Plujko et al., Nucl. Data Sheets 110, 3107 (2009)

[6] F. Becvar, Nucl. Inst. and Met. in Phys. Res. A 417, 434 (1998)

[7] A.B. Smith, P.R. Fields, A.M. Friedman, Phys. Rev. 104, 699 (1956)

[8] E. Nardi, A. Gavron, Z. Fraenkel, Phys. Rev. C 8, 2293 (1973)

[9] K. Skarsvag, Phys. Rev. C 22, 638 (1980)

[10] A. Chyzh, C.Y. Wu, E. Kwan, R.A. Henderson, J.M. Gostic, T.A. Bredeweg, R.C. Haight, A.C. Hayes-Sterbenz, M. Jandel, J.M. O Donnell et al., Phys. Rev. C 85, 021601 (2012)

[11] A.S. Vorobyev, V.N. Dushin, F.J. Hambsch, V.A. Jakolev, V.A. Kalinin, A.B. Laptev, B.F. Petrov, O.A. Shcherbakov, in Proceedings of the International Conference on Nuclear Data for Science and Technology (R. C. Haight et al., Santa Fe, 2004), Vol. AIP Conf. Proc. No. 769

[12] J.B. Wilhelmy, E. Cheifetz, R.C. Jared, S.G. Thompson, H.R. Bowman, J.O. Rasmussen, Phys. Rev. C 5, 2041 (1972)

[13] S. Mukhopadhyay, L.S. Danu, D.C. Biswas, A. Goswami, P.N. Prashanth, L.A. Kinage, A. Chatterjee, R.K. Choudhury, Phys. Rev. C 85, 064321 (2012)

[14] W. Hauser, H. Feshbach, Phys. Rev. 87, 366 (1952)

[15] A.J. Koning, S. Hilaire, S. Goriely, Talys 1.4 user manual (2011) 